\title{
Carbon lon Therapy: A Review of an Advanced Technology
}

\author{
Jung-in Kim ${ }^{1,2,3(\mathbb{D}}$, Jong Min Park ${ }^{1,2,3,4}$, Hong-Gyun $\mathrm{Wu}^{1,2,3,5,6(\mathbb{D})}$ \\ ${ }^{1}$ Department of Radiation Oncology, Seoul National University Hospital, ${ }^{2}$ Institute of Radiation Medicine, Seoul National University Medical \\ Research Center, ${ }^{3}$ Biomedical Research Institute, Seoul National University Hospital, Seoul, ${ }^{4}$ Robotics Research Laboratory for Extreme \\ Environments, Advanced Institute of Convergence Technology, Suwon, ${ }^{5}$ Department of Radiation Oncology, Seoul National University \\ College of Medicine, ${ }^{6}$ Cancer Research Institute, Seoul National University College of Medicine, Seoul, Korea
}

Received 29 May 2020

Revised 16 August 2020

Accepted 9 September 2020

Corresponding author

Hong-Gyun Wu

(wuhg@snu.ac.kr)

Tel: 82-2-2072-3177

Fax: 82-2-765-3317
This paper provides a brief review of the advanced technologies for carbon ion radiotherapy (CIRT), with a focus on current developments. Compared to photon beam therapy, treatment using heavy ions, especially a carbon beam, has potential advantages due to its physical and biological properties. Carbon ion beams with high linear energy transfer demonstrate high relative biological effectiveness in cell killing, particularly at the Bragg peak. With these unique properties, CIRT allows for accurate targeting and dose escalation for tumors with better sparing of adjacent normal tissues. Recently, the available CIRT technologies included fast pencil beam scanning, superconducting rotating gantry, respiratory motion management, and accurate beam modeling for the treatment planning system. These techniques provide precise treatment, operational efficiency, and patient comfort. Currently, there are $12 \mathrm{CIRT}$ facilities worldwide; with technological improvements, they continue to grow in number. Ongoing technological developments include the use of multiple ion beams, effective beam delivery, accurate biological modeling, and downsizing the facility.

Keywords: Carbon ion radiation therapy, Advanced techniques, Superconducting gantry, Effective beam delivery, Advanced beam modeling

\section{Introduction}

Carbon ion radiotherapy (CIRT) is an ion beam therapy for cancer treatment belonging to a family of particle therapies, such as protons, helium, and other ions. In 1946, Wilson [1] introduced the clinical use of particles. In his study, the biophysical rationale for proton particle therapy and beam delivery techniques was demonstrated. In the 1950s, high-energy accelerators were developed and utilized in clinical treatment. In 1954, Lawrence Berkeley National Laboratory (LBL) started particle therapy using protons [2]. Moreover, from 1975 to 1992, the LBL investigated various ion species, such as helium, carbon, and neon, and others, for ion beam therapy [3]; however, in 1992, it terminated all particle therapy investigations after trials with several particle species. In 1994, CIRT was initiated at the National Institute of Radiological Sciences (NIRS) in Japan using the world's first heavy ion accelerator complex (Heavy Ion Medical Accelerator in Chiba, HIMAC) [4]. NIRS adopted carbon ions among several types of ion species because of its high linear energy transfer (LET), which enables sufficient dose delivery within the target volume, in particular at the Bragg peak. Furthermore, carbon ions show high relative biological effectiveness (RBE) compared to conventional low-LET radiations in cell killing. Subsequently, in Germany, Gesellschaft für Schwerionenforschung (GSI) started CIRT in 1997, followed by the Heidelberg Ion Beam Therapy Center (HIT) in 2009. In Japan, Hyogo Ion Beam 
Medical Center (HIBMC) was the first proton and carbon facility established in 2001. In China, the Institute of Modern Physics (IMP) started clinical trials in 2006 and the Shanghai Proton Heavy Ion Center (SPHIC) started clinical application of proton and carbon beams in 2014. Due to the technological development at NIRS, in Japan, downsized carbon facilities were constructed at Gunma University Heavy Ion Medical Center (GHMC), SAGA Heavy Ion Medical Accelerator in Tosu (SAGA HIMAT), and Kanagawa Cancer Center (iROCK). GHMC, SAGA HIMAT, and iROCK commenced CIRT in 2010, 2013, and 2015, respectively. In Italy, the medical accelerator was installed for proton and carbon treatments at the National Center of Oncological Hadrontherapy (CNAO) in 2011, while in Austria, MedAustron started clinical studies in 2016. Recently, Osaka Center started CIRT in 2018. Currently, CIRT is available in 12 centers worldwide [5]. Moreover, there are two institutions under construction for CIRT in Yamagata, Japan, and Yonsei University, Korea, and several facilities are in the planning phase around the world.

By 2017, there were more than 20,000 patients who had undergone treatment with CIRT, mainly enrolled in phase I-II trials [6-8]. Japanese CIRT facilities conducted dose escalation and hypofractionation trials with a broad spectrum of indications. HIMAC employed a broad-beam delivery technique for irradiation field formation. Using scattering and orthogonal electromagnets, the accelerated carbon beam was broadened laterally, while using an exchangeable ridge filter, the beam range was adjusted to form a spread-out Bragg peak (SOBP) longitudinally. The SOBP was tailored to be tumor-shaped longitudinally using a range shifter and a custom-made range compensator and laterally using a multileaf collimator (MLC) or a custommade block $[9,10]$. Furthermore, the synchronized gating technique for moving target was applied to broad-beam delivery [11]. On the other hand, GSI adopted a pencil beam scanning (PBS) system for customized irradiation field. The accelerated carbon ion beam was scanned to form a thin Bragg peak layer with modulated intensity using orthogonal electromagnets. By changing beam energies, the layers of a variable SOBP were sequentially generated to fully conform to the tumor without additional devices [12]. Moreover, HIT, CNAO, and SPHIC adopted the PBS technology developed at GSI. HIT developed the first rotating gantry system for CIRT [13], which is important in multifield treatments.

CIRT has been established and evolved through the remarkable development and progress in technology and clinical studies. New technologies are being introduced for further improvement of the CIRT performance. The paper briefly reviews the ongoing technological developments.

\section{Characteristics of Carbon lon Therapy}

Recently, a growing interest in CIRT has been observed among several ion beam therapies due to its beneficial characteristics. Carbon ion beams have favorable dose distribution compared to photon and proton beams, providing a higher tumor control probability while minimizing the dose for the surrounding normal tissues. Therefore, we briefly review the physical and biological characteristics of carbon ion beams regardless of the technical aspects.

\section{Physical aspects}

For heavy charged particles such as carbon ions, the physically absorbed dose curve in the matter shows a slow initial increase with the penetration depth, then a steep rise to the maximum, known as the Bragg peak, followed by a sharp fall toward the end of the particles' range. The heavy charged ions have less lateral scattering than the light charged particles, such as electrons. Along the track of the traveling particle, the accelerated particles interact predominantly with the electrons in the matter by Coulomb's forces. The particle's range is determined by the energy of the incoming particles in response to the stopping power. The most deposited energy is at the end of the particle's range. The peak of dose distribution is a few millimeters wide, narrow, and sharp. Fig. 1 displays the depth dose distribution of various kinds of radiation in water [14], where the energy spread and range straggling affect the dose distribution. These phenomena depend on Coulomb's scattering and become smaller with increasing particle mass [15]. As regards the lateral distribution from the incident beam direction, the angular deflection by multiple scattering decreases with increasing charge and mass. Therefore, the lateral fall-off is more rapid in heavy ion beams than that in light ion beams 


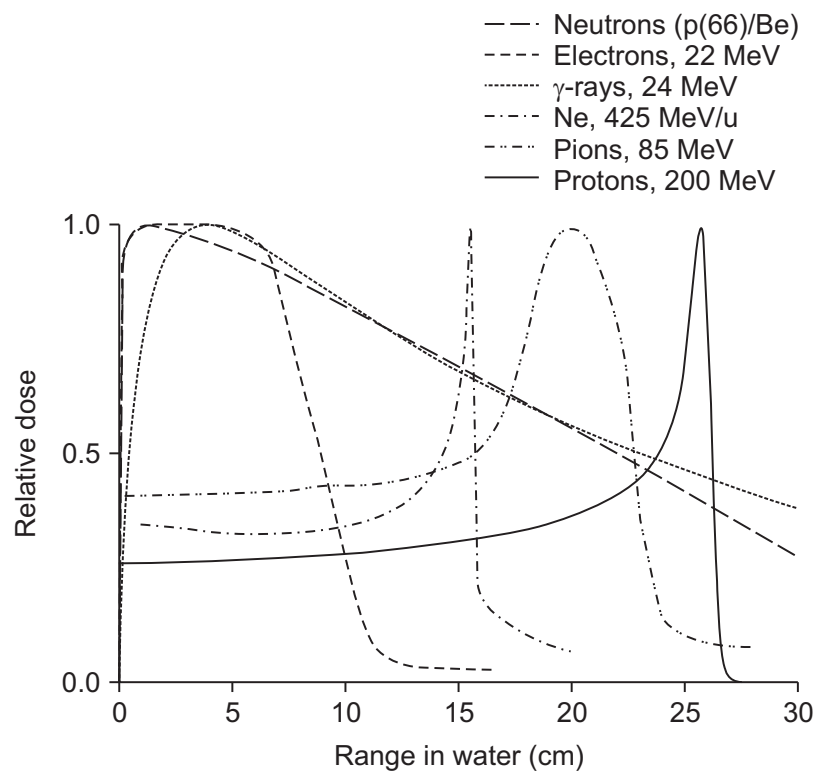

Fig. 1. Dose distributions as a function of depth in water for various clinical radiation beams [14].

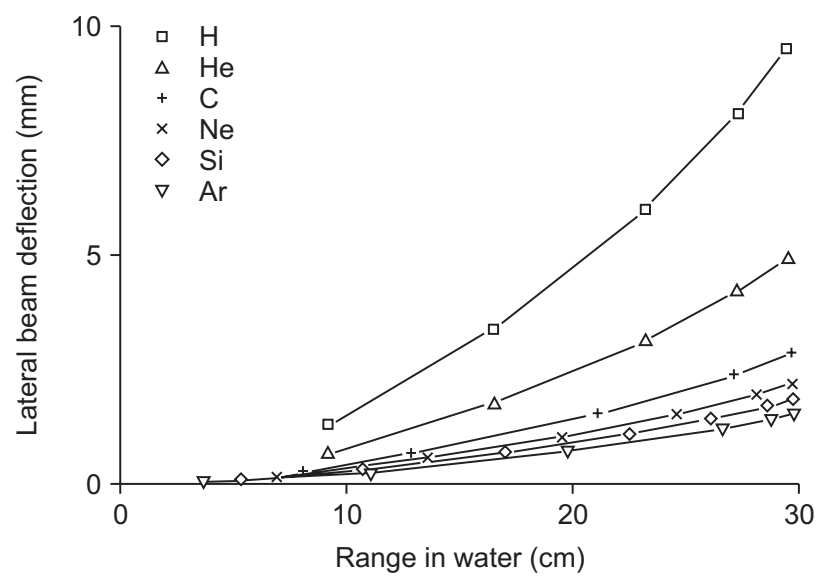

Fig. 2. Lateral beam deflection as a function of path length. Data from Lawrence Berkeley National Laboratory (LBL), Berkeley, USA [14].

and the degree of lateral sharpness (penumbra) for carbon ions is smaller than that of protons. Fig. 2 illustrates the lateral beam deflection with penetration depth for several ions. The effect for carbon is increased by about a factor of 3.5, compared to that for protons [14]. Therefore, CIRT offers a superior dose distribution due to its physical characteristics.

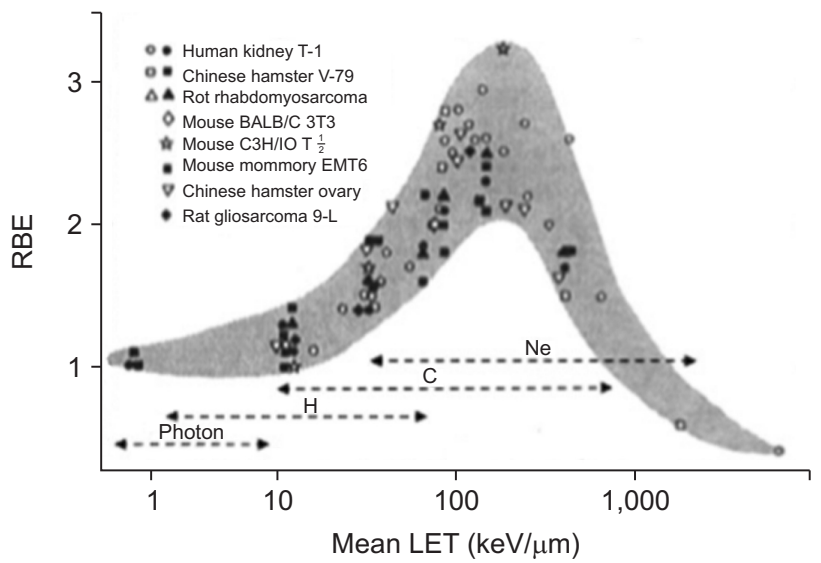

Fig. 3. Relative biological effectiveness (RBE) of ion beams as a function of linear energy transfer (LET) [17].

\section{Biological aspects}

Carbon ions are classified as high-LET radiation, resulting in dense ionization along their track. LET is defined as the energy transfer by an ionizing particle traveling through the matter. The stopping power of accelerated ions increases toward the end of their tracks, leading to more biological damage or an increase in RBE at the Bragg peak [16]. The RBE rises with increasing LET up to $100-200 \mathrm{keV} / \mu \mathrm{m}$ and decreases at higher LET values (Fig. 3) [17]. RBE is a complex entity depending on LET of the test radiation, physical dose, tumor type, tumor depth, and so forth. Generally, RBE or protons is 1.1, whereas RBE for carbon ions has been accepted to be in the 2-3.5 range. Another important phenomenon of the carbon ion beam is the oxygen enhancement ratio (OER), which is defined as the ratio of the isoeffect dose to hypoxic cells to that to aerobic cells. OER has a value of 1 for well-oxygenated tumors and values of up to 3 for hypoxic tumors for photons and protons. Tumors with low radiosensitivity against low-LET radiation are assumed to have a high proportion of hypoxic cells, poor reoxygenation, and high intrinsic repair. The OER reduction is achieved with increasing LET. Thus, high-LET carbon has considerably low OER and is more effective against hypoxic radioresistant tumors. Fig. 4 demonstrates different survival behaviors with ions and $\mathrm{X}$-rays according to the presence and absence of oxygen [18]. 


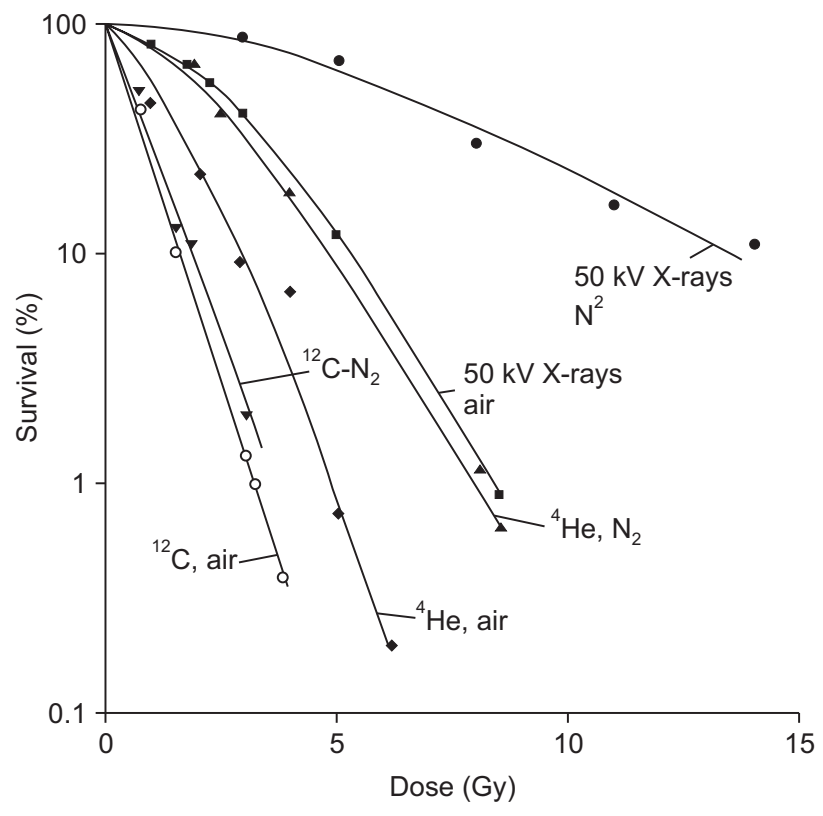

Fig. 4. Cell survival curves of human kidney T1 cells after irradiation with ions or X-rays in air or nitrogen atmosphere, respectively [18].

\section{Advanced Technologies for Carbon Ion Therapy}

Carbon ion beams provide potential advantages in terms of their physical and biological properties. Recent available technologies of CIRT are as follows: fast PBS, superconducting rotating gantry, respiratory motion management, and accurate beam modeling for TPS. These techniques may offer precise treatment, operational efficiency, and patient convenience. Furthermore, the emerging technological developments include the use of multiple ion beams, effective beam delivery, accurate biological modeling, and downsizing of facilities.

\section{Beam delivery}

Advanced beam delivery systems could enhance CIRT performance. Beam scanning, rotating gantry, irradiation parameters of the field size and dose rate, and multi-ion beams are technologies related to beam delivery which are briefly reviewed as follows.

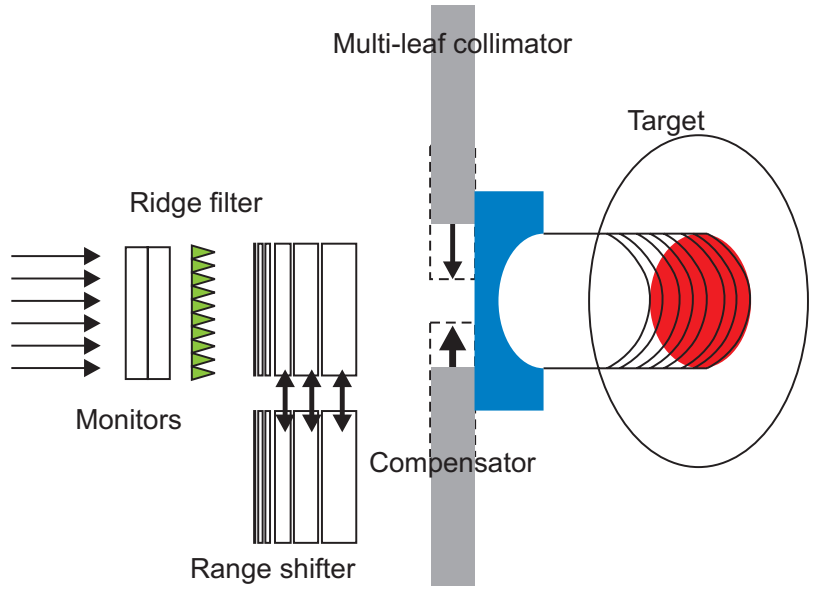

Fig. 5. Scheme of the principle of the layer-stacking method [19].

\section{1) Pencil beam scanning}

Accelerated ion beams with therapeutically relevant energy result in a narrow and sharp Bragg peak. For treating tumors of different sizes and shapes, the narrow peak should be broadened to cover the clinical target. Two methods have been introduced for this problem: a beam scattering method and a beam scanning method. Conventionally, a passive beam shaping device has been used to broaden the beam profile transversely and extend the Bragg peak dose from the distal to the proximal end of the target volume, respectively. Although conventional passive beam irradiation requires relatively simple treatment planning, there is one disadvantage which is the significantly excessive doses delivered to the normal tissues along the entrance to the target. Layer stacking was proposed to reduce this excessive dose and to achieve higher dose conformation with passive beam $[19,20]$. Fig. 5 illustrates the principle layer-stacking method. This technique uses a fine ridge filter, a range shifter, and MLC. The target is subdivided into regions with a 2.5 mm layer along the beam direction. By changing the MLC opening, the beam field size is defined to fit the respective subdivided regions. However, passive beam delivery techniques still have disadvantages as follows. The efficiency of beam utilization is low, $10 \%-30 \%$, resulting in a relatively large neutron contamination and high beam intensity; the beam shaping devices such as the compensator and MLC are required. Nevertheless, a PBS method still has more advantages than a broad-beam method as follows. For irregularly shaped tumors, the dose distribution is more confor- 
mal; the efficiency of beam utilization is almost $100 \%$; the amount of radiation shielding, thus, can be less than that in broad-beam method; secondary neutron can be significantly reduced. GSI developed the hybrid scanning system with a pencil beam [21]. Both HIT and CNAO used it in routine treatment [22,23]; however, PBS was not robust against the position and profile errors of the beam, and further, the risk of dose degradation increased by interplay with organ motion. Therefore, the PBS method was not generally applied for respiratory moving targets. In 2006, NIRS initiated a project to construct the New Particle Therapy Research Facility (NPTRF) for carbon ion beam scanning delivery. The scanning systems of NPTRF directly followed a developed and tested prototype [24]. The scanning speed of 100 $\mathrm{m} / \mathrm{s}$ was achieved on the isocenter plane painting each layer quickly [24]. In 2007, Furukawa et al. [25] proposed the respiratory phase-controlled rescanning method in order to apply PBS to the respiratory moving target. In 2012, the energy was rapidly changed one after another with a range shifter during beam delivery by multienergy extended-flattop extraction [26]. After the commissioning of 200 energy steps in 2015 [27], the full energy scanning mode without range shifting was introduced for clinical operation in 2017 [28]. Recent PBS methods have been developed with two sets of scanning magnets to scan the beam in a plane within the target volume. There are two ways to scan the beam spot: dynamic (or continuous) scanning method in a raster or line pattern, where the scan speed, beam intensity, or both are varied to produce the prescribed intensity distribution by treatment planning; discrete spot scanning method, where the beam is cut off between spots and the dose at each spot is varied to achieve the prescribed dose pattern. The continuous raster scanning is utilized to save the beam on/off time during beam movement between spot positions instead of discrete spot scanning. Pencil beam 3D scanning is an irradiation method where a pencil beam is laterally scanned to make a lateral irradiation field with orthogonal scanning dipole magnets and then longitudinally scanned by either a range shifter or energy change. The 3D scanning method incorporated with a rotating gantry can achieve higher treatment accuracy applying intensity-modulated particle therapy with multiple fields [29]. The advanced PBS technique will be further developed to achieve faster scanning speeds, smaller spot size, and more accurate spot positioning.

\section{2) Superconducting rotating gantry}

The rotating gantry can irradiate the tumor from several angles. Conventionally, the supine position is used because it minimizes the displacement of internal organs and is the most comfortable position for the patient. The gantry system offers more flexibility when selecting beam directions than the fixed beam. By choosing the most adequate beam incidence, the sensitive organs and the complex density heterogeneities can be avoided in the patient's body.

In 1990, the first proton beam radiotherapy facility with a rotating gantry was built by Loma Linda (Fermilab), whereas, In 2009 , the first facility with a $360^{\circ}$ rotating gantry for CIRT was constructed in HIT; its characteristics were as follows: length, $25 \mathrm{~m}$; radius, $6.5 \mathrm{~m}$; weight, about 600 tons. HIT used only normal conducting magnets for the gantry, whereas NIRS installed a second rotating gantry for CIRT in 2017 , which was the first rotating gantry with superconducting magnets with a significantly reduced size (length, $13 \mathrm{~m}$; radius, $5.5 \mathrm{~m}$ ) and weight (about 300 tons). The current developed superconducting rotating gantry is being installed at Yamagata University, Japan, with a length of approximately $25 \mathrm{~m}$, a radius of approximately $5.5 \mathrm{~m}$, and a weight of about 200 tons. By increasing the magnetic field strength produced by the superconducting magnet, the gantry size of $40 \%$ was achieved.

The most innovative feature for CIRT is the superconducting rotating gantry, allowing a compact small-sized and lightweight gantry design. Furthermore, when energy modulation is conducted upstream, the associated fast changes in magnet and on their power supplies. Recently, Kim et al. [30] presented a very compact gantry design for CIRT, where the gantry's length and radius were $9.74 \mathrm{~m}$ and $3.17 \mathrm{~m}$, respectively, reduced by using 5-T superconducting bending magnets for $\mathrm{C}^{6+}$ ion beam of $400 \mathrm{MeV} / \mathrm{u}$. It can transport an asymmetric beam and yields a beam with a full width at half maximum of $4 \mathrm{~mm}$ at the isocenter regardless of the rotating angle. Upstream parallel scanning covers a $20 \times 20 \mathrm{~cm}^{2}$ field size at the isocenter. 
Jung-in Kim, et al : Carbon lon Therapy: A Review of an Advanced Technology

\section{3) Irradiation parameters}

Clinically, field size and dose rate are important irradiation parameters. Field size is the distance between the 50\% points of the maximum dose value, measured along the line perpendicular to the beam central axis, on the isocenter plane in the air. The maximum field sizes for scanned beams of ions are currently smaller than those for passive beams because the high magnetic field requires fast deflection of the high-energy ion beams. The maximum field size for the scanned beam at HIT is $20 \times 20 \mathrm{~cm}^{2}$, whereas, at HIMAC, the maximum field sizes with the scanned beam are $22 \times 22 \mathrm{~cm}^{2}$ and $20 \times 20 \mathrm{~cm}^{2}$ for the fixed beam and gantry, respectively. Using translations of the treatment table may facilitate the treatment of larger fields. Clinically, a larger field size is required for CIRT, which can be increased by downsizing a scanning magnet, in other words, shortening the source-to-axis distance. The maximum field size of $25 \times 25 \mathrm{~cm}^{2}$ is being installed at Yamagata University, Japan.

The dose escalation studies of hypofractionated CIRT were conducted by the Japan group. At NIRS, Clinical trials were carried out with a hypofractionated schedule, and favorable results were obtained. The dose rate is another major parameter influencing the quality of treatment by determining the treatment time and affecting the radiobiological properties. Using passive beam methods, the typical dose rate required for treatment is about $5 \mathrm{GyE} /$ min (roughly equal to a physical dose of $2 \mathrm{~Gy}$ ) to $1 \mathrm{~L}$ volume corresponding to beam intensity of $10^{9}$ particles per second (pps), under a beam utilization efficacy of about $20 \%$ to $30 \%$. Therefore, in PBS, the beam intensity can be decreased because the beam utilization efficacy increases to almost $100 \%$. In the scanning beam methods, the typical dose rate required for treatment is about $2 \mathrm{~Gy} / \mathrm{min}$ to $1 \mathrm{~L}$ volume corresponding to around $10^{8} \mathrm{pps}$. The higher dose rate could be achieved by modifying the dose monitor and control system. The extracted particle number per second will increase by increasing the dose monitor dynamic range. However, for continuous beam scanning with a high dose rate (e.g., $4 \mathrm{~Gy} / \mathrm{min} / \mathrm{L}$ ), the amount of extra dose during beam moving between positions should be evaluated because the beam is not turned off.

\section{4) Multi-ion beams}

In the last few decades, proton and carbon ion particle therapies have been developed and their clinical applications have proved to be feasible and effective. Other ion beams, such as helium, oxygen, and neon, have been investigated and applied in treating tumors with different radiation sensitivities against different ion species. At LBL, helium and neon ions have been examined in clinical trials [31,32]. Between 1977 and 1994, around 2,000 patients were treated with helium ions using passive beam delivery at LBL [33]. Recent studies suggest that helium ions will be used as a potential treatment beam for further particle therapy improvement due to their favorable physical characteristics [34-37]. Helium ions show a low-dose fragmentation tail compared to carbon ions and exhibit less lateral beam spread and a higher LET than protons. From the biological point of view, helium RBE is closer to that of protons and certainly lower than that of carbon ions. HIT studied helium ion beams using measurements and Monte Carlo simulation in terms of dose distributions with single pencil beam, SOBP plans, and nuclear fragmentation [3740]. In 2020, the first European clinical program using helium ions by the raster-scanning method will be launched by HIT. Moreover, the beam delivery of multiple ion species has been investigated to maximize the therapeutic effects of charged particle beam in a single treatment session [41,42]. Inaniwa et al. [41] introduced the intensity-modulated composite particle therapy using two or more ion species in one treatment session, which enables the optimization of the dose and the LET distribution. The planning target volume (PTV) has higher LET than that of OARs while keeping the dose to PTV uniform. Recently, the European group developed the combined ion beam with constant RBE particle therapy in single field arrangements [43]; they reported that biophysically robust particle therapy treatments are feasible when combining heavy and light ions in a single treatment field, yielding uniform effective dose, RBE, and, in turn, physical dose distributions within the target. Through comprehensive dosimetric and in vitro study, multi-ion particle therapy will provide great clinical advantages. 


\section{Motion management}

Generally, intrafractional motion can blur dose distribution. Moreover, organ movement significantly affects dose deposition in particle therapy because the range of the ion beam can be changeable.

\section{1) Gating delivery and rescanning}

Respiratory motions are significantly important when treating moving target, especially in the treatment of lung cancer, which may be reduced in two primary ways as follows: (1) beam gating, where the beam is turned off after the target has moved out of the calculated treatment position; (2) repainting layers or treatment volume (i.e., repainting means to scan two or more times). In 2007, Furukawa et al. [25] developed a respiratory phase-controlled rescanning method to apply PBS to the respiratory moving target. A rescanning technique can be employed to average the effect of the irradiation at different breathing phases. This technique compensates for the interplay effects due to the residual motion of the target. Since rescanning does not involve any specific hardware, margins related to technical complexity will be low and comparable to stationary irradiation. The scanning speed is typically assumed to be sufficient using scanner magnets. The treatment control system mainly requires for rescanning. Gating requires a beam control system for pausing and resuming the beam based on the motion surrogate. Given the technical development and the current situation, motion mitigation reply on rescanning technique. However, studying the beam tracking method for motion mitigation can be a research topic to maximize target conformation and minimize treatment time. By selecting the appropriate motion mitigation technique, the best target coverage and conformation will be achieved with far less treatment time.

\section{Treatment planning}

Typically, treatment planning is a process to design radiation beams yielding the optimal balance between high dose conformation to the target and sparing of normal tissues. Treatment planning for carbon ions is similar to that of protons; the only major difference is the RBE variability.
RBE is variable as a function of particle type, incident energy, penetration depth, fraction dose, clinical endpoint, and so forth. In principle, the TPS should consider all these factors; however, only RBE modeling is briefly reviewed in this study. For a passive beam delivery system, the RBEweighted depth dose curves are stored as a beam library for the SOBP; these depth dose curves are scaled by the prescribed dose during dose calculation. This is useful for a well-defined clinical situation because a single fixed-range modulator is designed for a certain fractionation scheme, cell type, and endpoint. However, for example, if multiple fields are used or a dose per fraction is changed, the RBE will change. On the other hand, in a 3D scanning beam delivery system, to describe the respective depth dose curves, more general modeling is required, since nearly any arbitrary depth modulation can be achieved. Currently, GSI uses the local effect model (LEM) for the treatment planning of scanned beams, which uses microscopic features of the energy deposition of the ions around their tracks to explain the increased RBE and thus termed "track structure model." This model considers the effectiveness of the nuclear fragments and the dependencies of the resulting RBE on the applied dose, cell type, and endpoint. In 2011, the microdosimetric kinetic model (MKM) has been clinically introduced at NIRS which considers the dose dependence of RBE and the dependence of the RBE on the fragment spectrum produced by the primary ions [44]. The difference between MKM and LEM is the representation of the photon dose-response data. Generally, the LEM and MKM have been shown to be reasonable agreement with the in vitro measurements for a large range of ions. Both the LEM and the MKM and future treatment planning approaches should be validated with the steadily growing database of clinical experience with particles. The stochastic microdosimetric kinetic model has been developed for treatment planning of scanned helium, carbon, oxygen, and neon ion beams and their combinations [45].

Theoretical modeling of the biological effects of heavy ions is a challenging task because of the complexity and limited knowledge of the physical, chemical, and biological processes involved. The advanced biophysical models will provide improved treatment planning and guidance for further development to increase the application of ion beam therapy. 


\section{Conclusion}

Since 1970, CIRT has progressed and evolved rapidly in terms of technical delivery, indications, and efficacy. Particle therapy treatment delivery, treatment planning, and treatment quality are going through revolutionary advancements. These clinical, physical, and biological developments will significantly enhance the performance and reduce the cost of ion beam therapy, increasing its quality and availability in the medical care system. In the next decade, further improvements are expected in particle therapy's efficiency, robustness, and accuracy. More hospital-based, state-of-the-art particle therapy facilities will be built and increasing numbers of patients will be treated.

\section{Conflicts of Interest}

The authors have nothing to disclose.

\section{Availability of Data and Materials}

All relevant data are within the paper and its Supporting Information files.

\section{Author Contributions}

Conceptualization: Jung-in Kim and Hong-Guyn Wu. Data curation: Jong Min Park. Formal analysis: Jung-in Kim and Jong Min Park. Investigation: Hong-Guyn Wu. Methodology: Jung-in Kim and Jong Min Park. Supervision: HongGuyn Wu. Validation: Hong-Guyn Wu. Writing-original draft: Jung-in Kim. Writing-review \& editing: Jong Min Park and Hong-Guyn Wu.

\section{References}

1. Wilson RR. Radiological use of fast protons. Radiology. 1946;47:487-491.

2. Lawrence JH, Tobias CA, Born JL, McCombs RK, Roberts JE, Anger HO, et al. Pituitary irradiation with high-energy proton beams: a preliminary report. Cancer Res. 1958;18: $121-134$

3. Castro JR, Linstadt DE, Bahary JP, Petti PL, Daftari I, Col- lier JM, et al. Experience in charged particle irradiation of tumors of the skull base: 1977-1992. Int J Radiat Oncol Biol Phys. 1994;29:647-655.

4. Tsujii H, Mizoe JE, Kamada T, Baba M, Kato S, Kato H, et al. Overview of clinical experiences on carbon ion radiotherapy at NIRS. Radiother Oncol. 2004;73 Suppl 2:S41-S49.

5. Particle Therapy Co-Operative Group. Particle therapy facilities in clinical operation. Particle Therapy Co-Operative Group, 2018 [cited 2020 May 5]. Available from: https:// www.ptcog.ch/index.php/facilities-in-operation.

6. Kamada T, Tsujii H, Blakely EA, Debus J, De Neve W, Durante $\mathrm{M}$, et al. Carbon ion radiotherapy in Japan: an assessment of 20 years of clinical experience. Lancet Oncol. 2015; 16:e93-e100.

7. Pommier P, Lievens Y, Feschet F, Borras JM, Baron MH, Shtiliyanova A, et al. Simulating demand for innovative radiotherapies: an illustrative model based on carbon ion and proton radiotherapy. Radiother Oncol. 2010;96:243249.

8. Combs SE, Ellerbrock M, Haberer T, Habermehl D, Hoess A, Jäkel O, et al. Heidelberg Ion Therapy Center (HIT): initial clinical experience in the first 80 patients. Acta Oncol. 2010;49:1132-1140.

9. Kanai T, Endo M, Minohara S, Miyahara N, Koyama-ito $\mathrm{H}$, Tomura H, et al. Biophysical characteristics of HIMAC clinical irradiation system for heavy-ion radiation therapy. Int J Radiat Oncol Biol Phys. 1999;44:201-210.

10. Torikoshi M, Minohara S, Kanematsu N, Komori M, Kanazawa M, Noda K, et al. Irradiation system for HIMAC. J Radiat Res. 2007;48 Suppl A:A15-A25.

11. Minohara S, Kanai T, Endo M, Noda K, Kanazawa M. Respiratory gated irradiation system for heavy-ion radiotherapy. Int J Radiat Oncol Biol Phys. 2000;47:1097-1103.

12. Haberer T, Becher W, Schardt D, Kraft G. Magnetic scanning system for heavy ion therapy. Nucl Instrum Methods Phys Res Sect A. 1993;330:296-305.

13. Combs SE, Jäkel O, Haberer T, Debus J. Particle therapy at the Heidelberg Ion Therapy Center (HIT)- integrated research-driven university-hospital-based radiation oncology service in Heidelberg, Germany. Radiother Oncol. 2010;95:41-44.

14. Linz U. Physical and biological rationale for using Ions in therapy. Ion beam therapy. Berlin, Heidelberg: Springer; 
2012.

15. Chen GT, Castro JR, Quivey JM. Heavy charged particle radiotherapy. Annu Rev Biophys Bioeng. 1981;10:499-529.

16. Kraft G, Kraft-Weyrather W, Ritter S, Scholz M, Stanton J. Cellular and subcellular effect of heavy ions: a comparison of the induction of strand breaks and chromosomal aberration with the incidence of inactivation and mutation. Adv Space Res. 1989;9:59-72.

17. Chu W, Ludewigt B, Renner T. Instrumentation for treatment of cancer using proton and light-ion beams. Rev Sci Instrum. 1993;64:2055-2122.

18. Todd P. Fractionated heavy ion irradiation of cultured human cells. Radiat Res. 1968;34:378-389.

19. Kanai T, Kanematsu N, Minohara S, Komori M, Torikoshi M, Asakura H, et al. Commissioning of a conformal irradiation system for heavy-ion radiotherapy using a layerstacking method. Med Phys. 2006;33:2989-2997.

20. Futami Y, Kanai T, Fujita M, Tomura H, Higashi A, Matsufuji N, et al. Broad-beam three-dimensional irradiation system for heavy-ion radiotherapy at HIMAC. Nucl Instrum Methods Phys Res Sect A. 1999;430:143-153.

21. Kanematsu N, Endo M, Futami Y, Kanai T, Asakura H, Oka $\mathrm{H}$, et al. Treatment planning for the layer-stacking irradiation system for three-dimensional conformal heavy-ion radiotherapy. Med Phys. 2002;29:2823-2829.

22. Eickhoff H, Bar R, Dolinskii A, Haberer T, Schlitt B, Spiller $P$, et al. HICAT- The German hospital-based light ion cancer therapy project. Paper presented at: Proceedings of the 2003 Particle Accelerator Conference; 2003 May 12-16; Portland, USA. p. 694-698.

23. Borloni E, Rossi S. The CNAO project and the status of the construction. Paper presented at: Proceedings of NIRSCNAO Joint Symposium on Carbon Ion Radiotherapy; 2006 Nov 27-28; Milano, Italy.

24. Furukawa T, Inaniwa T, Sato S, Shirai T, Takei Y, Takeshita E,et al. Performance of the NIRS fast scanning system for heavy-ion radiotherapy. Med Phys. 2010;37:5672-5682.

25. Furukawa T, Inaniwa T, Sato S, Tomitani T, Minohara S, Noda K, et al. Design study of a raster scanning system for moving target irradiation in heavy-ion radiotherapy. Med Phys. 2007;34:1085-1097.

26. Inaniwa T, Furukawa T, Kanematsu N, Mori S, Mizushima $\mathrm{K}$, Sato S, et al. Evaluation of hybrid depth scanning for carbon-ion radiotherapy. Med Phys. 2012;39:2820-2825.

27. Hara Y, Furukawa T, Mizushima K, Inaniwa T, Saotome N, Tansho R, et al. Commissioning of full energy scanning irradiation with carbon-ion beams ranging from 55.6 to 430 $\mathrm{MeV} / \mathrm{u}$ at the NIRS-HIMAC. Nucl Instrum Methods Phys Res Sect B. 2017;406:343-346.

28. Furukawa T, Hara Y, Mizushima K, Saotome N, Tansho R, Saraya Y, et al. Development of NIRS pencil beam scanning system for carbon ion radiotherapy. Nucl Instrum Methods Phys Res Sect B. 2017;406:361-367.

29. Lomax A. Intensity modulation methods for proton radiotherapy. Phys Med Biol. 1999;44:185-205.

30. Kim J, Yoon M. Design of a compact gantry for carbon-ion beam therapy. Phys Rev Accel Beams. 2019;22:101601.

31. Lyman JT, Howard J. Dosimetry and instrumentation for helium and heavy ions. Int J Radiat Oncol Biol Phys. 1977;3: 81-85.

32. Phillips TL, Fu KK, Curtis SB. Tumor biology of helium and heavy ions. Int J Radiat Oncol Biol Phys. 1977;3:109-113.

33. Saunders W, Castro JR, Chen GT, Collier JM, Zink SR, Pitluck S, et al. Helium-ion radiation therapy at the Lawrence Berkeley Laboratory: recent results of a Northern California Oncology Group Clinical Trial. Radiat Res Suppl. 1985; 8:S227-S234.

34. Kempe J, Gudowska I, Brahme A. Depth absorbed dose and LET distributions of therapeutic $1 \mathrm{H}, 4 \mathrm{He}, 7 \mathrm{Li}$, and $12 \mathrm{C}$ beams. Med Phys. 2007;34:183-192.

35. Kantemiris I, Karaiskos P, Papagiannis P, Angelopoulos A. Dose and dose averaged LET comparison of ${ }^{1} \mathrm{H},{ }^{4} \mathrm{He},{ }^{6} \mathrm{Li}$, ${ }^{8} \mathrm{Be},{ }^{10} \mathrm{~B},{ }^{12} \mathrm{C},{ }^{14} \mathrm{~N}$, and ${ }^{16} \mathrm{O}$ ion beams forming a spread-out Bragg peak. Med Phys. 2011;38:6585-6591.

36. Tessonnier T, Marcelos T, Mairani A, Brons S, Parodi K. Phase space generation for proton and carbon ion beams for external users' applications at the Heidelberg Ion Therapy Center. Front Oncol. 2015;5:297.

37. Krämer M, Scifoni E, Schuy C, Rovituso M, Tinganelli W, Maier A, et al. Helium ions for radiotherapy? Physical and biological verifications of a novel treatment modality. Med Phys. 2016;43:1995.

38. Marafini M, Paramatti R, Pinci D, Battistoni G, Collamati F, De Lucia E, et al. Secondary radiation measurements for particle therapy applications: nuclear fragmentation produced by ${ }^{4} \mathrm{He}$ ion beams in a PMMA target. Phys Med Biol. 
2017;62:1291-1309.

39. Tessonnier T, Mairani A, Brons S, Sala P, Cerutti F, Ferrari A, et al. Helium ions at the Heidelberg Ion Beam Therapy Center: comparisons between FLUKA Monte Carlo code predictions and dosimetric measurements. Phys Med Biol. 2017;62:6784-6803.

40. Tessonnier T, Mairani A, Brons S, Haberer T, Debus J, Parodi K. Experimental dosimetric comparison of ${ }^{1} \mathrm{H},{ }^{4} \mathrm{He},{ }^{12} \mathrm{C}$ and ${ }^{16} \mathrm{O}$ scanned ion beams. Phys Med Biol. 2017;62:39583982.

41. Inaniwa $\mathrm{T}$, Kanematsu N, Noda K, Kamada T. Treatment planning of intensity modulated composite particle therapy with dose and linear energy transfer optimization. Phys Med Biol. 2017;62:5180-5197.

42. Krämer M, Scifoni E, Schmitz F, Sokol O, Durante M. Overview of recent advances in treatment planning for ion beam radiotherapy. Eur Phys J D. 2014;68:306.

43. Kopp B, Mein S, Dokic I, Harrabi S, Böhlen TT, Haberer T, et al. Development and validation of single field multi-ion particle therapy treatments. Int J Radiat Oncol Biol Phys. 2020;106:194-205.

44. Inaniwa T, Kanematsu N, Matsufuji N, Kanai T, Shirai T, Noda K, et al. Reformulation of a clinical-dose system for carbon-ion radiotherapy treatment planning at the National Institute of Radiological Sciences, Japan. Phys Med Biol. 2015;60:3271-3286.

45. Inaniwa T, Suzuki M, Hyun Lee S, Mizushima K, Iwata Y, Kanematsu N, et al. Experimental validation of stochastic microdosimetric kinetic model for multi-ion therapy treatment planning with helium-, carbon-, oxygen-, and neonion beams. Phys Med Biol. 2020;65:045005. 